\title{
With Gottlieb beyond Gottlieb: The Role of Epigenetics in Psychobiological Development
}

\author{
Vanessa Lux* \\ Center for Literary and Cultural Research Berlin, Berlin, Germany
}

\begin{abstract}
The emerging field of molecular epigenetics studies relatively stable changes in genetic activity that are not due to changes in the DNA sequence. Initial research results indicate a functional role for epigenetic mechanisms in neuron development and neuronal cell function. However, concepts that integrate these findings in an overall theory of psychobiological development have yet to be developed. Gilbert Gottlieb's probabilistic epigenesis and his scheme of psychobiological development provide an ideal starting point for this effort. Based on a modified version of Gottlieb's scheme of psychobiological development, this study conceptualizes epigenetic mechanisms as molecular underpinnings mediating interactions between neural and genetic activity levels. Three different functional contexts of epigenetic mechanisms in neuronal cells are identified: genomic, developmental, and synaptic. Finally, consequences for a model of psychobiological development and implications for future research in molecular epigenetics are discussed.
\end{abstract}

Keywords

Epigenetics, psychology, development, model, probabilistic epigenesis

In 1991, Gilbert Gottlieb proposed a scheme of psychobiological development based on his concept of probabilistic epigenesis (Gottlieb, 1991a, p. 6; see Fig. 1). In the same article he also expressed the "hope that the immense gap between molecular biology and developmental psychology will one day be filled with facts, as well as with valid concepts" (Gottlieb 1991a, p. 10). Since then, molecular biology has seen immense progress in research methods, with sequencing techniques, microarray technology, and computational statistics providing the means to study inner-cell processes on a molecular level. Gottlieb himself actively collected empirical evidence from this line of research in late publications (Gottlieb, 1998, 2003, 2007). He was especially interested in mechanisms regulating gene activity, cell-cell communication and gene-environment interaction. The emerging field of molecular epigenetics is dedicated to the uncovering of these mechanisms.

\footnotetext{
*Address for correspondence

Dr. Vanessa Lux, Center for Literary and Cultural Research Berlin, Schützenstr. 18, 10117 Berlin, Germany. E-mail: vanessa.lux@fuberlin.de
}

Molecular epigenetics will produce and has already produced some noteworthy facts to fill the gap between molecular biology and developmental psychology. However, concepts which allow us to integrate these facts into theories of psychobiological development have yet to be developed. Gottlieb's probabilistic epigenesis and his scheme of psychobiological development provide an ideal starting point for this effort, but in light of recent molecular epigenetic findings, further elaboration is needed. The aim of this article is to propose a modified version of Gottlieb's model which conceptually integrates probabilistic epigenesis and molecular epigenetics. It also proposes consequences for a model of psychobiological development and implications for molecular epigenetics, thus taking Gottlieb beyond Gottlieb.

\section{Developmental vs. Molecular "Epigenetics"}

The term epigenetics was coined by Conrad $\mathrm{H}$. Waddington in 1942 (Waddington, 1942; see also Holliday, 2002; Jablonka \& Lamb, 2002, p. 82). It is not by chance that the term is philologically and conceptually 


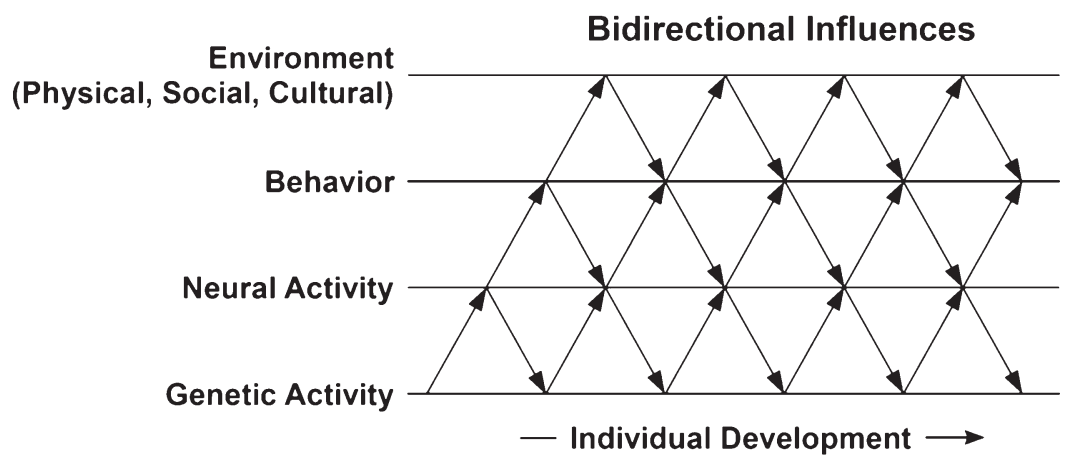

Figure 1. Gilbert Gottlieb's metatheoretical model of probabilistic epigenesis (according to Gottlieb, 1992).

reminiscent of epigenesis, the rival theory to preformationism in early embryology from Aristotle to the 19th century (Waddington, 1942; see also Van Speybroeck, De Waele, \& Van de Vijver, 2002). Waddington's aim was to theoretically and empirically reconcile the synthesis of genetics and evolutionary theory with the earlier embryology (Huxley, 1956, p. 807; see also Haig, 2012, p. 14). Waddington was especially interested in the question of stability of organ development under varying developmental conditions. He rejected simple genetic predetermination and, instead, proposed a model of developmental pathways ("creodes") characterized by complex self-stabilizing mechanisms of gene-gene and gene-environment interactions which he illustrated with the image of the "epigenetic landscape" (see Waddington, 1957, pp. 26-38).

In contrast to Waddington's developmental epigenetics, the new field of molecular epigenetics focuses on "mitotically and/or meiotically heritable changes in gene function that cannot be explained by changes in DNA sequence" (Riggs, Martienssen, \& Russo, 1996, p. 1; similarly Holliday, 1994; Wu \& Morris, 2001). DNA methylation and acetylation, histone modification and RNA interference, their stability during cell division and their sensitivity to environmental cues such as nutrition or stress are studied. These molecular epigenetic mechanisms have been interpreted as additional inheritance systems complementing genetic inheritance (e.g. Jablonka \& Lamb, 2005), and, due to their environmental sensitivity, they are discussed as proof of the inheritance of acquired characteristics, with fundamental consequences for our understanding of heredity and evolution (Jablonka \& Raz, 2009).

However, most of the effects of molecular epigenetic mechanisms that have thus far been observed occur in soma cells during the lifespan of an individual and are not transmitted to the next generation. Some authors even argue that the criterion of mitotic or meiotic transmission is too tight to capture all epigenetic mechanisms of gene regulation (e.g. Bird, 2007). This is most obvious in neuronal cells as these cells usually do not undergo further mitosis. Molecular epigenetics of neuronal cells or "neuroepigenetics" is therefore defined as the study of those mechanisms "which acutely or persistently modify transcription in cells, irrespective of their position in the cell cycle and which do not mutate the genome" (Sultan \& Day, 2011; similarly Gräff, Kim, Dobbin, \& Tsai, 2011).

With its sole focus on gene expression, molecular epigenetics has more or less abandoned the broader developmental perspective of its origins. So the question arises how today's molecular epigenetic findings can be reintegrated into a developmental framework as represented by Gottlieb's probabilistic epigenesis.

\section{Gottlieb's Probabilistic Epigenesis}

As with Waddington's developmental epigenetics, Gottlieb's probabilistic epigenesis proposes a model of how the phenotype is brought into being during ontogeny. And like Waddington, Gottlieb emphasizes the complexity and plasticity of developmental processes and the influence of non-genetic factors. However, Gottlieb's model of probabilistic epigenesis differs essentially from Waddington's developmental pathways. Gottlieb himself pointed out that Waddington's pathways are still strongly predetermined by interaction among genes and therefore by the genotype (Gottlieb, 1991a, p. 5). Gottlieb focused instead on the role of experience and the developmental environment as crucial for genetic activity. The differences in the way genes and genetic activity are conceptualized within each of the models may be due in 
part to different research objectives: While Waddington studied morphogenesis and organ development on a genetic and tissue level, Gottlieb studied the development of sensory functions and behavioral patterns on a behavioral and neural level. However, profound conceptual differences result from it: For Waddington, further morphological structures such as organs emerge from interactions between genes, and genegene interactions are able to stabilize the developmental pathway against environmental influences. For Gottlieb, genes are embedded in an overall developmental system which influences their activity.

Accordingly, Gottlieb conceptualized ontogenetic development as a multi-level dynamic system with hierarchical but bidirectional and coactional relationships within and between all levels and at all points of development. In 1991, he illustrated his perspective with a model scheme of psychobiological development including four levels of development: genetic activity, neural activity, behavior, and environment (Gottlieb, 1991a). One year later he added further specifications to the environmental level, differentiating between physical, social, and cultural aspects of environment (Gottlieb, 1992, see Fig. 1).

The notion of bidirectionality and coaction originates from his studies on acoustic development in avian embryos, based on natural observation and experimental work. Gottlieb demonstrated how the preference for the maternal assembly call after hatching, e.g. in mallard duck chicks, depends on pre-hatching experiences of embryonic species-specific vocalization, either in the form of self-vocalization or of vocalization of other chicks (Gottlieb, 1991b, 1999; see also Gottlieb, 1971, 1976). He later concluded in more general terms that "neural (and other) structures begin to function before they are fully mature and this activity, whether intrinsically derived ('spontaneous') or extrinsically stimulated (evoked), plays a significant role in the development process" (Gottlieb, 2007, p. 2). According to his own account (Gottlieb, 1992, pp. 158-159), Gottlieb was strongly influenced by the interactionist views of developmental psychologists Zing-Yang Kuo (e.g. Kuo, 1976), Theodore Christian Schneirla (e.g. Schneirla, 1960), and Daniel S. Lehrmann (e.g. Lehrman, 1970) in the conceptualization, execution, and interpretation of his experimental work. His own metatheoretical model of development may be interpreted as integration and further radicalization of their emphasis on the developmental relevance of pre- and early post-natal environment, self-stimulation and reciprocal interactions between different levels of development.
Besides bidirectionality and coaction, Gottlieb's probabilistic epigenesis is built on two further developmental principles: the principle of equifinality and the principle of non-linear or probabilistic causality. Referring to Hans Driesch and his cell separation experiments in sea urchins, Gottlieb interpreted equifinality as the ability of "developing organisms of the same species" to "reach the same endpoint via different developmental pathways" (Gottlieb, 2003, p. ${ }^{\circ}$; see Driesch, 1905, p. 213). Equifinality is a necessary condition for the fourth principle: the principle of non-linear or probabilistic causality. With the label "probabilistic," Gottlieb positions his model of development against unidirectional "predetermined epigenesis" models (Gottlieb, 2007, p. 5). For Gottlieb the term "probabilistic" holds the notion of non-linear and, more importantly, nondeterministic causal relationships between different levels in the developmental system, which results in "some degree of indeterminacy" of the developmental process and outcome (Gottlieb, 2003, p. 14; see also Gottlieb, 2007, p. 2). However, as Jaan Valsiner points out, "making sense of probability is the key issue" (Valsiner, 2007a, p. 835) in Gottlieb's concept. Based on biological field theory interpretations of causality, Valsiner specifies Gottlieb's concept of probability as one of "indeterministically deterministic" relationships between developmental levels depending on structural possibilities of the developmental system (propensity notion of probability) (Valsiner, 2007a).

As one could conclude from his later writings (e.g. Gottlieb, 1998, 1999, 2003, 2007), the four principles of Gottlieb's model-bidirectionality, coaction, equifinality and probability (non-predetermined causality) -have been accepted among colleagues to be relevant for the three upper levels of the scheme, neuronal activity, behavior and environment, but not for the genetic level. Until recently, Francis Crick's central dogma of molecular biology - genetic activity functions unidirectionally from DNA to RNA to protein and never in reverse (Crick, 1970) — prevailed in genetics and dominated the perception of genetics in psychology. But according to Gottlieb's model, the interactions do not stop at the cell membrane: "genes are not exempt from influences at other levels of analysis but are, in fact, dependent upon them for initiating and terminating their activity" (Gottlieb, 2007, p. 2). As a psychologist and developmental biologist, Gottlieb could not proof the validity of his model for the genetic level. One attempt to include measures of protein synthesis from the auditory and visual parts of mallard duck brains as measures of genetic activity, a study Gottlieb executed together 
with neurologists George Paulson and Stanley Apple as early as 1965, failed because data analysis of the protein synthesis was never completed and the results were never published (Gottlieb, 2003, p. 8). Nevertheless, Gottlieb collected a wide range of evidence of interactions between genetic activity and neural, sensory and environmental dimensions of development to support his perspective (Gottlieb, 1998, 2007; see also Valsiner, 2007b).

The growing evidence from molecular epigenetic research showing the ubiquity and key role of epigenetic mechanisms in the regulation of genetic activity due to higher level functions, and especially in response to neural activity, supports Gottlieb's model. Moreover, Gottlieb's model of development, illustrated by him with the scheme of multi-level bidirectional psychobiological development (see Fig. 1), proposes a framework to fit molecular epigenetic findings into an overall developmental theory. For this, however, a few modifications of the model are needed.

\section{Integrating Molecular Epigenetics into the Framework of Probabilistic Epigenesis}

From a developmental perspective, epigenetic mechanisms understood as mechanisms involved in the unfolding and emergence of new structures and functions during development are present at all levels of development. In contrast, the mechanisms studied in molecular epigenetics are located in the individual cell and between cells. To our current knowledge, on the molecular level in and between cells they contribute to a vast number of developmental processes including cell differentiation, organ development, motor learning etc. If we look at psychological development, molecular epigenetic mechanisms active in neuronal cells are those of most interest. Here, molecular epigenetic mechanisms serve as mediating mechanisms between the neuronal cell's genetic activity and its own neural activity as well as that of surrounding cells and connected neurons. One important characteristic of Gottlieb's model is its focus on relationships between levels, as this is where development takes place (Valsiner, 2007b). Molecular epigenetic mechanisms in neuronal cells are strongly involved in such between-level interactions in the daily cell metabolism of neurons, as well as in key developmental processes in psychobiological development.

Figure 2 shows a modified version of Gottlieb's scheme which illustrates how research results from molecular epigenetic mechanisms active in neuronal cells could be integrated into the framework of probabilistic epigenesis. Within this modified scheme, molecular epigenetic mechanisms are not assigned an additional level but instead are placed between the neural and the genetic activity level to highlight their role as between-level mechanisms. Further modifications of the scheme follow an earlier modified version by Valsiner (2007b, p. 838) replacing the environment level with a level of "higher psychological functions." The level of "higher psychological functions" accounts for intentionality and the ability to produce meaning through language and cultural artifacts unique in humans. Its hierarchical relationship with motor and sensory activity has been shown extensively in psychology, e.g. in studies on impulse control (Mischel, Shoda, \& Rodriguez, 1989) and on the influence of prior expectation on sensory functions (Bruner \& Postman, 1949). In addition, the term "behavior" is used in multiple ways in psychology, meaning everything from reflex-driven activity to the conduct of everyday life and cultural traditions based on decision making and planning. Therefore, to increase discriminatory power between the behavior level and the level of higher psychological functions the behavior level is relabeled "sensory and motor activity" level. Also, the relationship between levels will be, in most cases, cyclical (see Valsiner, 2007b), and is best understood as a feed-forward loop-represented by the horizontal arrows in Fig. 2.

Furthermore, the "environment" is placed as overall back-layer of development. While Gottlieb's placement of the environment at the upper level of the developmental system puts a stronger emphasis on the hierarchical relationship between the levels and highlights the constitutive role of environment (social, cultural, physical) for individual development, it implies that the causal relationships between the environment and other levels of the developmental system are always mediated by behavior. This is certainly true for a wide range of individual-environment interactions central to psychobiological development such as sensory-motor coordination and, on another level, the development of consciousness and higher psychological functions. However, the physical dimensions of the environment, such as gravity, temperature, radiation or chemicals may also directly influence the genetic and the neural activity level (see also Johnston \& Edwards, 2002). Further qualitative and structural specifications of the environmental back-layer are necessary to account for the societal nature of the human developmental system, and its social and institutional subunits (fam- 


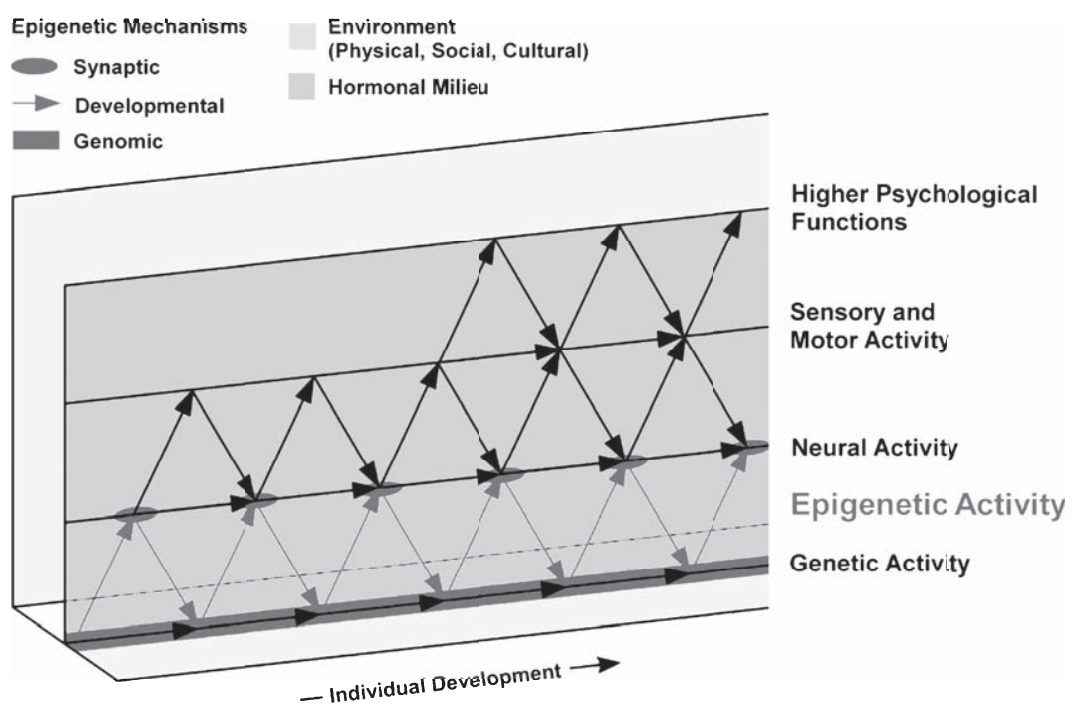

Figure 2. Modified model of psychobiological development with epigenetic mechanisms. Three functional contexts of epigenetic mechanisms are distinguished: genomic, developmental, and synaptic.

ily, school, workplace) have been demonstrated to be highly relevant to psychosocial and even psychobiological development (e.g. Lerner, 1999; Lerner \& Lerner, 1989). The scheme presented here is simplified in this regard to emphasize the role of molecular epigenetic activity within psychobiological development.

Finally, I highlight a second back-layer representing the "hormonal milieu" within the body. Hormones not only play a key role in the regulation of the general body metabolism, but also regulate organ development (e.g. Torday, 2003; Torday, Ihida-Stansbury, \& Rehan, 2009) including early brain development (Tsutsui, 2012). The hormonal milieu represents a key dimension of the inner body environment in psychobiological development (see also Gottlieb, 2003). This milieu mediates between physiological and psychobiological development, although the exact processes are not entirely known.

Based on this model, three different functional contexts of molecular epigenetic mechanisms in psychobiological development can be identified from current research results in epigenetics: genomic, developmental, and synaptic (see Fig. 2).

\section{Genomic Mechanisms}

Most epigenetic mechanisms at the genomic or chromatin level regulate neuron-specific gene expression through DNA methylation and histone modification (Houston et al., 2013; Moore, Le, \& Fan, 2013). However, some of these epigenetic mechanisms function as proper genomic mechanisms. They serve either as genomic repair mechanisms (Barzilai, Biton, \& Shiloh, 2008) or maintain low expression levels of pro-apoptotic genes (Morrison et al., 2006). Especially histone deacetylase-1 (HDAC1) seems to be involved in the maintenance of neuron-specific suppression of cell-cycling genes (Kim et al., 2008) facilitating neuron-specific gene expression and longevity.

\section{Developmental Mechanisms}

DNA de/methylation and histone modification, both influencing the spatial and biochemical structure of DNA binding sites and their reactivity to transcription factors, regulate the differentiation of neuronal stem cells into neurons, astrocytes and oligodendrocytes (Soldati et al., 2012; see also Gräff et al., 2011; Olynik \& Rastegar, 2012). For example, during neuron development the removal of the repressor element1 silencing transcription factor (REST) molecule, known to suppress transcription of neuron-specific genes in non-neuronal cells from neuron specific gene sites, is accompanied by increased histone acetylation, residue-specific histone methylation, and decreased DNA methylation, causing local relaxation of the chromatin structure and enabling transcription of neuron specific genes (Gräff et al., 2011). The underlying epigenetic regulatory complex is reported to be sensitive to specific stimuli including membrane depolarization (Ballas, Grunseich, Lu, Speh, \& Mandel, 2005) and to non-specific developmental conditions such 


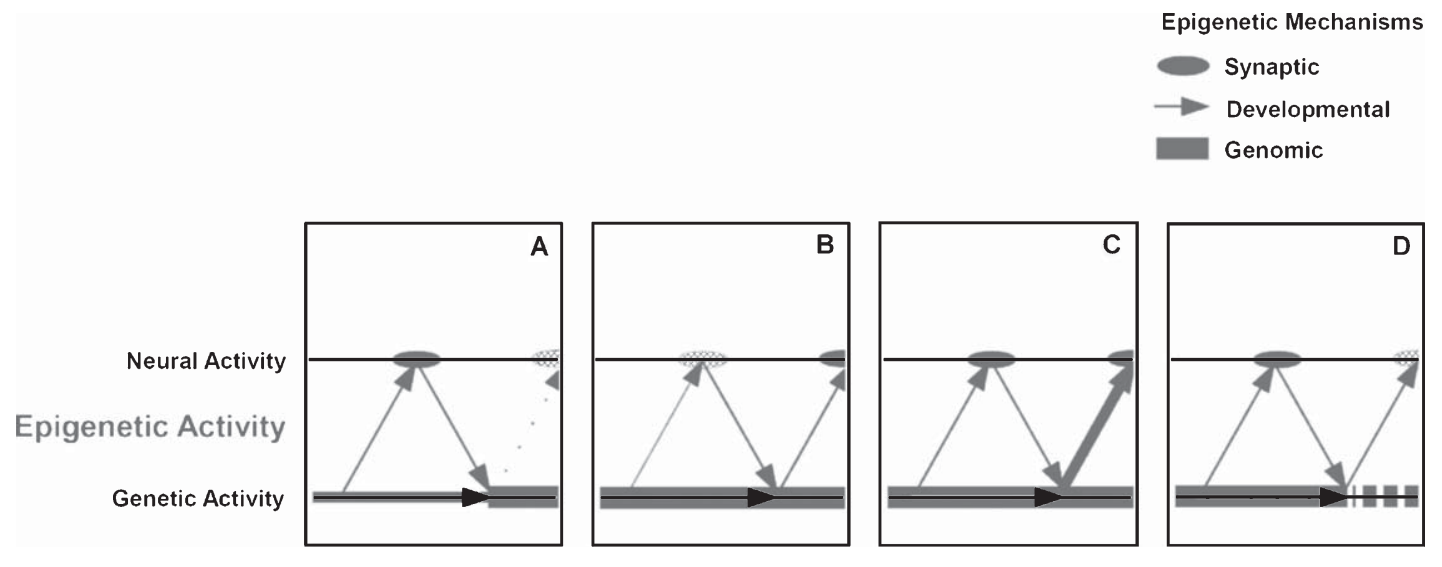

Figure 3. Epigenetic mechanisms may block (A), facilitate (B) or enhance (C) interaction between neural activity and genetic activity. Epigenetic mechanisms functioning as synaptic and genomic mechanisms may act as developmental mechanism (D).

as prenatal choline availability (Mehedint, Niculescu, Craciunescu, \& Zeisel, 2010). This indicates a multiphasic and bidirectional process at play involving interaction between genetic and neural activity levels as well as environmental conditions in early neuronal development. Furthermore, chromatin modifications are involved in the regulation of axon and dendrite growth (Trakhtenberg \& Goldberg, 2012), and DNA de/methylation is discussed as a mechanism regulating hippocampal adult neurogenesis sensitive to neural activity (Ma et al., 2009). In addition, in animal models epigenetic mechanisms have been shown to be sensible to behavioral changes in early development. One of the findings of most interest for a model of psychobiological development is that, in rats and mice, the long-term consequences of early-life stress for physiological stress response are mediated by epigenetic mechanisms (Franklin et al., 2010; Meaney \& Szyf, 2005).

\section{Synaptic Mechanisms}

DNA methylation and histone modification facilitate and maintain synaptic plasticity and function (Feng, Fouse, \& Fan, 2007; Sultan \& Day, 2011). For example, Nelson et al. reported that in vitro DNA methyltransferase inhibition decreased the frequency of miniature excitatory postsynaptic potentials exchanged between hippocampal postmitotic neurons, and that this effect is regulated by DNA methylation, e.g. at the brain-derived neurotrophic factor (BDNF) promoter I site (Nelson, Kavalali, \& Monteggia, 2008). BDNF is involved in the growth and differentiation of neurons and synapses and probably has functional relevance in long-term memory formation. Changes in synaptic transmission and BDNF promoter methylation depended on background neuronal activity in the cell cultures under study, demonstrating synaptic regulation of gene expression through epigenetic mechanisms. Furthermore, initial studies point to an additional role for RNA interference, the interaction of micro RNA molecules with DNA, messenger RNA or enzymes regulating protein synthesis, in synaptic plasticity (see Smalheiser, 2012; Sultan $\&$ Day, 2011). These molecular epigenetic mechanisms sensitive to synaptic signals, and especially the interplay of histone modification and DNA de/methylation, are interpreted as potential molecular underpinnings of memory formation (Feng et al., 2007; Gräff et al., 2011; Levenson \& Sweatt, 2005; Sultan \& Day, 2011), although the exact processes are yet unknown.

The modified version of Gottlieb's model (illustrated in Fig. 2) enables us not only to clearly distinguish these three functional contexts-genomic, developmental, and synaptic — but also to further hypothesize their interplay when it comes to psychobiological development (illustrated in Fig. 3). It proposes a scheme to functionally categorize single molecular epigenetic mechanisms for their overall functional role in psychobiological development. However, a few consequences for our understanding of psychobiological development as well as implications for future molecular epigenetic research arise from this union of probabilistic epigenesis and molecular epigenetics.

\section{Conclusions}

The new field of molecular epigenetics studies changes in gene expression that are not related to changes in 
the DNA sequence. For research on psychobiological development, molecular epigenetics findings in neuronal cells are of especially high interest. Gottlieb's probabilistic epigenesis proposes a framework that allows us to fruitfully integrate current epigenetic findings into an overall theory of psychobiological development, and to identify and evaluate possible contributions of molecular genetics to the problem of "how" genotype, contexts and experience "cooperate" in ontogenetic development-one of the core issues in Developmental Science (Scheithauer, Niebank, \& Gottlieb, 2007, p. 15). Within the modified version of Gottlieb's model of psychobiological development, molecular epigenetic mechanisms in neuronal cells are conceptualized as mechanisms mediating between neural and genetic activity. Based on this model, three functional contexts of molecular epigenetic mechanisms in neuronal cells-genomic, developmental, and synaptic_-are distinguished.

\section{Consequences for a Model of Psychobiological Development}

The observed interactions in molecular epigenetics have consequences for an overall model of psychobiological development. Most importantly, the finding that histone modification, DNA de/methylation, and RNA interference in neuronal cells mediate between neural and genetic activity shows that bidirectionality is present at all levels of psychobiological development including the genetic level. This confirms bidirectionality as a key principle of psychobiological development in general. Neural activity may in fact block, facilitate, and enhance genetic activity through molecular epigenetic mechanisms (see Fig. 3, A-C), as the examples of neuronal cell development, synaptic reactivity and memory formation outlined above illustrate. By enabling bidirectionality, epigenetic mechanisms represent a possible molecular basis of interactions between structural and functional dimensions of neuron development and neural activity. Two examples from current molecular epigenetic research illustrate this: First, HDAC1 and histone deacetylase-2 (HDAC2) regulate synaptic development in early neuron development. Akhtar et al. (2009) reported that in mice an induced decrease of HDAC1 and HDAC2 levels caused excitatory synapse maturation and modest increase in synapse numbers. In contrast, in mature neurons a decrease in HDAC2 levels alone caused basal excitatory neurotransmission. The authors proposed that HDAC1 and
HDAC2 interact as "developmental switch" which controls synapse maturation and function dependent on the maturational state of the neuron. This means, enzymatic processes regulating epigenetic mechanisms involved in neuron development and those maintaining synaptic function are interrelated indicating a possible fine tuning mechanism of early gene expression profiles due to developed neuronal activity. Second, activitydependent adult neurogenesis depends on Gadd45b (Growth arrest and DNA-damage-inducible protein 45 beta) expression. Ma et al (2009) showed that Gadd45b is required for selective DNA demethylation of functional binding sites of genes involved in the expression of proteins critical for adult neurogenesis, including BDNF and fibroblast growth factor. Thus, Gadd45b expression connects neural activity to genetic activity relevant for neurogenesis via molecular epigenetic activity (selective DNA demethylation). Integrating molecular epigenetic knowledge into a model of psychobiological development therefore allows reconciling structural and functional dimensions of neural development and neural networks. Furthermore, the ubiquity of epigenetic mechanisms in neuronal cells supports the importance of coaction of developmental levels in psychobiological development.

With regard to functional contexts, the role of epigenetic mechanisms goes beyond neuronal cell development over the life course. Molecular epigenetic mechanisms are as crucial for general neural activity as they are for development. However, while molecular epigenetic mechanisms functioning as genomic and synaptic mechanisms are primarily involved in regulating or maintaining a given state of neuronal cell activity, they may sometimes function as developmental mechanisms (see Fig. 3-D). For example, dysregulation of epigenetic control mechanisms and accumulation of aberrant epigenetic marks in neuronal cells due to aging are discussed as representing a molecular mechanism underlying age-related cognitive dysfunctions (Sweatt, 2010). From the perspective of developmental systems theory this strongly supports the ideas that (1) developmental processes rely on processes maintaining a current state of activity and (2) a current developmental stage has to be permanently maintained or reproduced (e.g. Gottlieb, 1976; Oyama, 1985).

To capture the complexity of multi-level interactions of the developmental system at a certain time-point, Timothy Johnston and Laura Edwards proposed a systemic network model of psychobiological function (Johnston \& Edwards, 2002). Although subsumed under the more general terms "protein synthesis" 
and "intracellular biochemistry," some molecular epigenetic mechanisms regulating gene expression are integrated into this model (Johnston \& Edwards, 2002, p. 28). Johnston and Edward's model impressively demonstrates the complexity of interactions at the neural activity level in relation to the development of non-neural structures and other developmental levels (physical environment, behavior, genetic activity). In comparison, the model of psychobiological development illustrated in Figure 2 focuses on the interactions between the genetic and the neural activity level mediated by epigenetic activity. It also preserves the emphasis on hierarchy of levels and inter-level interaction as well as the time axis of development from Gottlieb's original scheme, thereby highlighting the overall functional significance of molecular epigenetic mechanisms in psychobiological development.

\section{Implications for Molecular Epigenetic Research}

The modified model of psychobiological development (Fig. 2) has consequences for molecular epigenetics when used as theoretical framework to interpret molecular epigenetic data. For example, from a developmental perspective the role of epigenetic changes can be conceptualized as reducing and channeling variability instead of producing variability — as it may look like from a focus on DNA sequences and their varying functions regulated by molecular epigenetic mechanisms. In addition, from a developmental systems perspective it has to be assumed that these developmental constraints (e.g. in neuronal cell differentiation) must be actively maintained throughout the life of an individual (cell). This implicates that molecular epigenetics should not only study changes of gene expression but also the stability of these changes and how they are maintained.

Furthermore, based on the model of psychobiological development proposed here, further dynamics of epigenetic mechanisms may be hypothesized and tested in molecular epigenetic research. For example, it seems likely that, at given moments, the additional direct interaction of molecular epigenetic mechanisms may facilitate or even accelerate development. Epigenetic research focusing on such interactions at critical periods in development promises to be especially fruitful.

Based on the principle of equifinality, it may be assumed that there are different pathways for most developmental mechanisms. For molecular epigenetic research, this implies searching for additional backup mechanisms and feedback loops when discovering a new mechanism. Here, the principle of probability rather than causality provides a framework within which to interpret the interplay of fuzziness and structural dependence of epigenetic activity made visible in molecular epigenetic research. Both principles, however, imply that, due to possible inter-individual variability in developmental mechanisms, research has to focus on individuals and individual cells instead of populations (an all-in-one instead of a one-in-all approach—see also Gottlieb, 2003). The use of individual cell strains or genetically modified organisms raised under controlled conditions may propose a middle ground. It is therefore not surprising that epigenetic studies using these methods are producing the most promising results at the moment. However, regarding the role of social interaction and individual experiences in brain development and learning, to control developmental conditions may be difficult in studies on psychobiological development. Accordingly, generalization of studies is limited to individuals or individual populations.

To sum up, conceptualizing epigenetic mechanisms as catalytic functions embedded within a system of feedback loops and organized as partial open systems based on probability (non-predetermined causality) instead of predetermined causality, and to assume equifinality (or at least multiplicity of pathways), bidirectionality and coaction at all levels of development enables us, but also obliges us to describe and test current emerging assumptions on the molecular underpinnings of psychobiological development. The modified version of Gottlieb's model of psychobiological development described above proposes a promising framework within which to integrate current and future molecular epigenetic findings and to stimulate future studies. It also proposes an alternative to the metaphor of the "epigenetic code" currently emerging in scientific and popular literature which once again squeezes molecular biology findings into a framework of conceptual reductionism.

\section{Acknowledgments}

This work was made possible by funding of the Federal Ministry of Education and Research Germany. I would like to thank my colleagues at the Center for Literary and Cultural Research Berlin as well as Jaan Valsiner for fruitful discussions and helpful comments on an earlier version of this paper. 


\section{References}

Akhtar, M. W., Raingo, J., Nelson, E. D., Montgomery, R. L., Olson, E. N., \& Kavalali, E. T. et al. (2009). Histone deacetylases 1 and 2 form a developmental switch that controls excitatory synapse maturation and function. The Journal of Neuroscience, 29, 82888297.

Ballas, N., Grunseich, C., Lu, D. D., Speh, J. C., \& Mandel, G. (2005). REST and its corepressors mediate plasticity of neuronal gene chromatin throughout neurogenesis. Cell, 121, 645-657.

Barzilai, A., Biton, S., \& Shiloh, Y. (2008). The role of the DNA damage response in neuronal development, organization and maintenance: DNA Damage Responses and Neurological Disease. DNA Repair, 7, 1010-1027.

Bird, A. (2007). Perceptions of epigenetics. Nature, 447, 396-398.

Bruner, J. S., \& Postman, L. (1949). On the perception of incongruity; a paradigm. Journal of Personality, 18, 206-223.

Crick, F. (1970). Central Dogma of Molecular Biology. Nature, 227, 561-563.

Driesch, H. (1905). Der Vitalismus als Geschichte und als Lehre. Natur- und kulturphilosophische Bibliothek: III. Leipzig: Verlag von Johann Ambrosius Barth.

Feng, J., Fouse, S., \& Fan, G. (2007). Epigenetic regulation of neural gene expression and neuronal function. Pediatric Research, 61, 58R-63R.

Franklin, T. B., Russig, H., Weiss, I. C., Gräff, J., Linder, N., Michalon, A., Vizi, S., \& Mansuy, I. S. (2010). Epigenetic transmission of the impact of early stress across generations. Biological Psychiatry, 68, 408-415.

Gottlieb, G. (1971). Development of species identification in birds: An inquiry into the prenatal determinants of perception. Chicago: University of Chicago Press.

Gottlieb, G. (1976). The roles of experience in the development of behavior and the nervous system. In G. Gottlieb (Eds.), Neural and behavioral specificity (pp. 25-54). New York: Academic Press.

Gottlieb, G. (1991a). Experiential canalization of behavioral development: Theory. Developmental Psychology, 27, 4-13.

Gottlieb, G. (1991b). Experiential canalization of behavioral development: Results. Developmental Psychology, 27, 35-39.

Gottlieb, G. (1992). Individual development and evolution: The genesis of novel behavior. New York: Oxford University Press.

Gottlieb, G. (1998). Normally occurring environmental and behavioral influences on gene activity: From central dogma to probabilistic epigenesis. Psychological Review, 105, 792-802.

Gottlieb, G. (1999). Probabilistic epigenesis and evolution. 1999 Heinz Werner lecture series: Vol. 23. Worcester, Mass: Clark University Press.

Gottlieb, G. (2003). Probabilistic epigenesis of development. In J. Valsiner, \& K. J. Connolly (Eds.), Handbook of developmental psychology (pp. 3-17). London: Sage Publications.

Gottlieb, G. (2007). Probabilistic epigenesis. Developmental Science, 10, 1-11.

Gräff, J., Kim, D., Dobbin, M. M., \& Tsai, L-H. (2011). Epigenetic regulation of gene expression in physiological and pathological brain processes. Physiological Reviews, 91, 603-649.

Haig, D. (2012). Commentary: The epidemiology of epigenetics. International Journal of Epidemiology, 41, 13-16.
Holliday, R. (1994). Epigenetics. An overview. Developmental Genetics, 15, 453-457.

Holliday, R. (2002). Epigenetics comes of age in the twenty first century. Journal of Genetics, 81, 1-4.

Houston, I., Peter, C. J., Mitchell, A., Straubhaar, J., Rogaev, E., \& Akbarian, S. (2013). Epigenetics in the human brain. Neuropsychopharmacology, 38, 183-197.

Huxley, J. (1956). Epigenetics. Nature, 177, 807-809.

Jablonka, E., \& Lamb, M. J. (2002). The changing concept of epigenetics. Annals of the New York Academy of Sciences, 981, 82-96.

Jablonka, E., \& Lamb, M. J. (2005). Evolution in four dimensions: Genetic, epigenetic, behavioral, and symbolic variation in the history of life. Cambridge, Mass: MIT Press.

Jablonka, E., \& Raz, G. (2009). Transgenerational epigenetic inheritance: prevalence, mechanisms, and implications for the study of heredity and evolution. The Quarterly Review of Biology, 84, 131-176.

Johnston, T., \& Edwards, L. (2002). Genes, interactions, and the development of behavior. Psychological Review, 109, 2634.

Kim, D., Frank, C. L., Dobbin, M. M., Tsunemoto, R. K., Tu, W., Peng, P. L. et al. (2008). Deregulation of HDAC 1 by p25/Cdk5 in neurotoxicity. Neuron, 60, 803-817.

Kuo, Z.-Y. (1976). The dynamics of behavior development: An epigenetic view (New enlarged edition). New York: Plenum Press.

Lehrman, D. (1970). Semantic and conceptual issues in the naturenurture problem. In L. R. Aronson, E. Tobach, J. S. Rosenblatt, \& D. Lehrman (Eds.), Development and evolution of behavior. Essays in memory of T. C. Schneirla (pp. 17-52). San Francisco: Freeman.

Lerner, R. M. (1999). Dialectics, developmental contextualism, and the further enhancement of theory about puberty and psychosocial development. In R. M. Lerner \&, J. V. Lerner (Eds.), Theoretical foundations and biological bases of development in adolescence (pp. 308-330). New York: Garland.

Lerner, R. M., \& Lerner, J. V. (1989). Organismic and social contextual bases of development: The sample case of adolescence. In W. Damon (Ed.), Child development today and tomorrow (pp. 69-85). San Francisco: Jossey-Bass.

Levenson, J. M., \& Sweatt, J. D. (2005). Epigenetic mechanisms in memory formation. Nature Reviews, 6, 108-118.

Ma, D. K., Jang, M. H., Guo, J. U., Kitabatake, Y., Chang, M. L., PowAnpongkul, N. et al. (2009). Neuronal activity-induced Gadd45b promotes epigenetic DNA demethylation and adult neurogenesis. Science, 323, 1074-1077.

Meaney, M. J., \& Szyf, M. (2005). Maternal care as a model for experience-dependent chromatin plasticity? Trends in Neurosciences, 28, 456-463.

Mehedint, M. G., Niculescu, M. D., Craciunescu, C. N., \& Zeisel, S. H. (2010). Choline deficiency alters global histone methylation and epigenetic marking at the $\operatorname{Re} 1$ site of the calbindin 1 gene. The FASEB Journal, 24, 184-195.

Mischel, W., Shoda, Y., \& Rodriguez, M. L. (1989). Delay of gratification in children. Science, 244, 933-938.

Moore, L. D., Le, T., \& Fan, G. (2013). DNA methylation and its basic function. Neuropsychopharmacology, 38, 23-38.

Morrison, B. E., Majdzadeh, N., Zhang, X., Lyles, A., BasselDuby, R., Olson, E. N., et al. (2006). Neuroprotection by histone deacetylase-related protein. Molecular and Cellular Biology, 26, 3550-3564. 
Nelson, E. D., Kavalali, E. T., \& Monteggia, L. M. (2008). Activitydependent suppression of miniature neurotransmission through the regulation of DNA methylation. The Journal of Neuroscience, 28, 395-406.

Olynik, B. M., \& Rastegar, M. (2012). The genetic and epigenetic journey of embryonic stem cells into mature neural cells. Frontiers in Genetics, 3, 81.

Oyama, S. (1985). The ontogeny of information: Developmental systems and evolution Cambridge, Mass: Cambridge University Press.

Riggs, A. D., Martienssen, R. A., \& Russo, V. E. A. (1996). Introduction. In V. E. A. Russo, A. D. Riggs, \& R. A. Martienssen (Eds.), Cold Spring Harbor monograph series: Vol. 32. Epigenetic mechanisms of gene regulation (pp. 1-4). Plainview, NY: Cold Spring Harbor Laboratory Press.

Scheithauer, H., Niebank, K., \& Gottlieb, G. (2007). To See an Elephant: Developmental Science. International Journal of Developmental Science, 1, 6-22.

Schneirla, T. C. (1960). Instinctive behavior, maturation-Experience and development. In B. Kaplan, \& S. Wapner (Eds.), Perspectives in psychological theory - Essays in honor of Heinz Werner (pp. 302-334). New York: International Universities Press.

Smalheiser, N. R. (2012). The search for endogenous siRNAs in the mammalian brain. Experimental neurology, 235, 455-463.

Soldati, C., Bithell, A., Johnston, C., Wong, K. Y., Teng, S. W., Beglopoulos, V. et al. (2012). Repressor element 1 silencing transcription factor couples loss of pluripotency with neural induction and neural differentiation. Stem Cells, 30, 425-434.

Sultan, F. A., \& Day, J. J. (2011). Epigenetic mechanisms in memory and synaptic function. Epigenomics, 3, 157-181.

Sweatt, J. D. (2010). Epigenetics and cognitive aging. Science, 328, 701-702.

Torday, J. S. (2003). Parathyroid hormone-related protein is a gravisensor in lung and bone cell biology. Advances in Space Research, 32, 1569-1576.
Torday, J. S., Ihida-Stansbury, K., \& Rehan, V. K. (2009). Leptin stimulates Xenopus lung development: evolution in a dish. Evolution \& Development, 11, 219-224.

Trakhtenberg, E. F., \& Goldberg, J. L. (2012). Epigenetic regulation of axon and dendrite growth. Frontiers in Molecular Neuroscience, 5,24 .

Tsutsui, K. (2012). Neurosteroid biosynthesis and action during cerebellar development. Cerebellum, 11, 414-415.

Valsiner, J. (2007a). Gilbert Gottlieb's theory of probabilistic epigenesis: Probabilites and realities in development. Developmental Psychobiology, 49, 832-840.

Valsiner, J. (2007b). Probabilistic epigenesis as the central nondogma of development. European Journal of Developmental Science, 1, 101-122.

Van Speybroeck, L., De Waele, D., \& Van de Vijver, G. (2002). Theories in early embryology: Close connections between epigenesis, preformationism, and self-organization. Annals of the New York Academy of Sciences, 981, 7-49.

Waddington, C. H. (1942). The epigenotype. Endeavour, 1, 18-20.

Waddington, C. H. (1957). The strategy of the genes: A discussion of some aspects of theoretical biology. London: George Allen \& Unwin.

Wu, C.-T., \& Morris, J. R. (2001). Genes, genetics, and epigenetics: A correspondence. Science, 293, 1103-1105.

\section{Bio Sketch}

Vanessa Lux, $\mathrm{PhD}$, psychologist, is currently working as post-doctoral researcher in the interdisciplinary project, "Cultural Factors of Inheritance," at the Center for Literary and Cultural Research in Berlin. Her research focuses on the significance of epigenetic knowledge for developmental psychology and for the concept of psychological trauma. 